\title{
DEL OTRO LADO DEL ATLÁNTICO: \\ LAS AFROAMERICANAS Y SU LUCHA POR LA DOBLE IGUALDAD
}

TO THE OTHER SIDE OF THE ATLANTIC:

THE AFRICAN AMERICANS AND THEIR FIGHT FOR DOUBLE EQUALITY

\begin{abstract}
Ariadna Royo Herrera
Doctoranda Universitat Jaume I

RESUMEN

Las reivindicaciones por la igualdad legal y social de la población negra, canalizadas en el movimiento por los derechos civiles, fueron el marco de la década de los sesenta para los Estados Unidos. No sólo la cuestión racial estuvo presente entre esta población, las afroamericanas que participaban directamente comenzaron a ser conscientes del sexismo hacia ellas por parte de la sociedad blanca y su misma comunidad. La unión en torno a la congregación fue impulsada como defensa contra el racismo, al mismo tiempo se promovían modelos familiares y roles de género tradicionales. En la misma década, emerge un nuevo feminismo crítico con la sociedad del momento, pero impulsor de un único modelo para sus reivindicaciones; lo cual dificultaba la conexión interracial, al vivir problemáticas y demandas diferentes para cada grupo de mujeres.

Palabras Clave: racismo, patriarcado, feminismo, movimiento por los derechos civiles.

\section{ABSTRACT}

The demands for legal and social equality of the black population, channeled in the civil rights movement, was the framework of the 1960s for the United States. Not only the racial issue was present among this population, the African-Americans who participated directly began to be aware to the sexism towards them by the White society and their own community. The union around the congregation was promoted as a defense against racism, at the same time promoting family models and traditional gender roles. In the same decade, a new feminism emerged critical with the society of the momento, but driving a single model for its demands; which hindered the interracial connection, living different problems and demands for each group of women.
\end{abstract}

Keywords: racism, patriarchy, feminism, civil rights movement. 


\section{SUMARIO}

1.- Introducción. 2.- Lucha por la ciudadanía. 3.- La Segunda Ola: un único modelo de mujer. 4.- Jane Crow, mecanismos de control patriarcal. 5.- Bibliografía.

\section{Introducción}

El nacimiento de nuevas ideologías y movimientos sociales, que impulsan una sociedad crítica consigo misma y las instituciones gubernamentales, fue el marco de la década de 1960 a nivel global. El desarrollo de estas nuevas corrientes se caracterizó por implicar directamente a la ciudadanía-desde manifestaciones a asambleas-, por estar impulsadas desde diferentes focos, como fueron las universidades o las organizaciones civiles. La respuesta de los gobiernos a estas nuevas demandas fueron dispares, todo dependía de la capacidad de diálogo y asimilación de la necesidad de transformaciones internas que tuviera cada uno de ellos. Por ello, el contexto particular de cada país y las demandas particulares de cada grupo implicado fueron decisivos para comprender la evolución de esta década, y sobre todo vislumbrar por qué, a pesar de encontrar ciertas similitudes, no se puede hablar de un movimiento uniforme. Esto no descarta la influencia entre colectivos o naciones, como fueron Francia -principal referente a nivel europeo- o Estados Unidos.

Si se comparan los escenarios políticos, económicos y sociales de ambos países se encuentran diferencias significativas que otorgaron un carácter particular a los movimientos sociales de cada nación. En primer lugar, el fin de la Segunda Guerra Mundial implicó la reconstrucción de Europa y el establecimiento de nuevos gobiernos, en el caso de Francia destacó la presidencia de Charles de Gaulle'; mientras que Estados Unidos se encontraba intacto y convertido en la primera potencia mundial.

En segundo lugar, el crecimiento económico estadounidense estuvo acompañado por el despegue de la industria y la creación de nuevas industrias como la aeroespacial, así como un éxodo rural ocasionado por la mecanización del campo. Una situación que afectaba sobre todo a la población negra, que se traslada a los ghettos de las grandes ciudades industriales del norte y oeste del país. La esperanza por conseguir mejorar su nivel de vida pronto se encontró con el muro de la segregación, que impedía el progreso de esta población al recortar sus derechos y libertades. Por el contrario, en Francia encontramos el aumento del

1 Charles de Gaulle fue una de las principales figuras de la Segunda Guerra Mundial, sobre todo en el proceso de liberación de la Francia ocupada, con la creación, en el exilio, del movimiento de la Francia Libre. También formó parte del gobierno provisional de la República hasta 1946 y posteriormente presidió la República francesa entre 1958 y 1969. 
desempleo -especialmente juvenil- o la precariedad laboral. Pero, en el caso del país galo, afectando a toda la sociedad en su conjunto y no a una parte de la misma -una minoría racial-, como sucedía en la estadounidense.

Por último, la creciente tensión entre los/as afroamericanos/as, quienes estaban cansados de la hipocresía de su gobierno al luchar en Europa por la democracia, pero manteniendo en su territorio la opresión y el control sobre esta población por medio de la segregación. El nuevo espíritu de lucha fue canalizado por diferentes organizaciones que desembocaron en el movimiento por los derechos civiles. Una corriente iniciada a mediados de la década de los cincuenta, pero que no alcanzó su auge hasta la siguiente.

La celebración de las elecciones de 1960 fue otro punto a tener en cuenta, ya que los candidatos Richard Nixon y John F. Kennedy ofrecían dos posturas claramente diferenciadas. Nixon mantenía un discurso conservador basado en el anticomunismo, la religión y la unidad familiar; por el contrario, Kennedy se presentó como el candidato que recogía el espíritu joven y el clima de cambio que se estaba gestando. Bajo el término de contracultura, se englobó todo este nuevo pensamiento opuesto a la ideología impulsada por el gobierno y la sociedad norteamericanos hasta el momento; que fomentaba el consumismo desmedido, el materialismo, el miedo al comunismo o el mantenimiento de la hipocresía sexual, entre algunos de los puntos más destacados.

Movimientos tan dispares como el ecologismo, el feminismo, el hippie, la lucha del colectivo LGTB o por los derechos civiles de la población negra y nativa se incluyen dentro de la denominada contracultura, al presentar una ruptura contra el statu quo de la sociedad estadounidense del momento. A pesar de este punto en común, las diversas corrientes tuvieron un desarrollo y actuación diferentes dependiendo de sus objetivos y de sus ideologías particulares. Pero sin implicar una desconexión entre movimientos, como fue en Estados Unidos la lucha de los/as afroamericanos/as y la Segunda Ola del feminismo e incluso a nivel internacional el hippie en el Mayo francés.

\section{Lucha por la ciudadanía}

El vínculo entre la lucha de los/as afroamericanos/as por obtener la igualdad -tanto legal como social- y la feminista ha tenido una larga trayectoria dentro de Estados Unidos. Los inicios de esta unión se remontan a principios del siglo XIX con los grupos abolicionistas, compuestos y dirigidos principalmente por mujeres blancas de clase media-alta; quienes luchaban por la emancipación de los/as esclavos/as, pero sin ser realmente conscientes de qué suponía ser discriminado/a hasta la Convención Mundial Contra la Esclavitud celebrada 
en Londres (1840). Este momento marcó el inicio del sufragismo en Estados Unidos, por medio de Elizabeth Cady Stanton y Lucretia Mott, representantes de la Sociedad Estadounidense Antiesclavista, quienes fueron excluidas de las intervenciones y vetadas por su sexo. Un suceso considerado el impulsor de la Convención de Seneca Falls ${ }^{2}$ (1848), en la cual se planteó el debate sobre la discriminación de las mujeres y el sufragio femenino por primera vez en Estados Unidos.

En un primer momento, el abolicionismo y el sufragismo estuvieron estrechamente vinculados al formar, muchos de sus miembros, parte de ambos movimientos. No obstante, esta situación cambió a partir de 1863 con la aprobación de la Ley de Emancipación que otorgaba la libertad a los negros. También implicaba la ciudadanía para esta población y, por consiguiente, posibilitaba el voto al hombre negro. Ante esta posibilidad, algunas se sintieron amenazadas, al incluirse al varón negro -mayormente analfabeto y en situación de pobreza- en el poder político, ya que pensaban que serían más despóticos que sus homólogos blancos, como se recoge a continuación:

But in a narrow view of the question as a matter of feeling between classes, when Mr. Downing puts the question to me, are you willing to have the colored man enfranchised before the woman, I say, no; I would not trust him with all my rights; degraded, oppressed himself, he would be more despotic with the governing power than even our Saxon rulers are. I desire that we go into the kingdom together, for individual and national safety demand that not another man be enfranchised without the woman by his side (Stanton, 1887: 214) .

Las palabras de Stanton recogen este miedo al mantenimiento de la superioridad masculina -comprendida como poder social, político y económico-, al prolongarse en los hombres negros. La lucha por la liberación de la población negra pasaba a estar enfrentada al sufragio femenino. "Todo indica que ella estaba decidida a impedir que las personas negras [...] experimentaran un progreso si éste no significaba que las mujeres blancas pudieran disfrutar de los beneficios inmediatos que contenía tal progreso» (Davis, 2005: 78). Si apostaban por el sufragio, les permitiría alcanzar el poder político, considerado como el objetivo principal para la obtención de la igualdad entre sexos; mientras que si mantenían

\footnotetext{
2 La convención finalizó con la firma de La Declaración de Sentimientos -documento basado en la Declaración de Independencia-, en la cual se exponen las limitaciones de las mujeres estadounidenses y la discriminación que sufrían a diario.

3 Pero en una visión estrecha de la pregunta como una cuestión de sentimiento entre clases, cuando el Sr. Dowing me plantea la pregunta, ¿está dispuesta a tener al hombre negro con derecho al voto frente a la mujer? Mi respuesta es que no. No le confiaría mis derechos a un hombre degradado y oprimido que sería más despótico de lo que jamás hayan sido nuestros gobernantes anglosajones. Pido que vayamos juntos al reino, porque la seguridad individual y nacional exige que ningún otro hombre sea liberado sin la mujer a su lado. [Todas las traducciones del inglés son de la autora]
} 
el apoyo a la lucha de la población negra, pronto las fuerzas políticas -compuestas exclusivamente por hombres- no permitirían el avance de ninguna de ellas. Una premisa aceptada por las sufragistas, por lo que decidieron apostar primero por sus propios derechos.

Asimismo, Henry Ward Beecher ${ }^{4}$ llegó a afirmar que era más importante conseguir el voto femenino -entendido sólo para las blancas- que lograrlo para los hombres negros. Pues, al ser mujeres cultas y provenientes de entornos socioculturales elevados y presentarlas como madres/ciudadanas, que impulsan valores cívicos y morales en los futuros ciudadanos, eran mejores representantes de los valores cívicos que los varones negros.

Además, la creciente tensión entre ambos movimientos alcanzó tal punto que Stanton y otras compañeras llegaron a utilizar argumentos racistas o el término Sambo, que sirve para recoger todo un conjunto de estereotipos -desde estúpidos, bárbaros, ladrones, etcétera. La misma Stanton afirmaba: «In fact, it is better to be the slave of an educated White man, than of a degraded, ignorant black one» (Stanton, 1887: 94). Una afirmación utilizada para acusar a los afroamericanos de no preocuparse por la situación de las mujeres negras, quienes según Stanton estarían mejor siendo esclavas de hombres blancos que de sus congéneres.

Una línea de pensamiento que no fue apoyada por todo el movimiento sufragista. Abby Kelly Foster ${ }^{5}$ consideraba que la emancipación no implicaba inmediatamente el fin de la opresión económica y la equiparación social para los antiguos esclavos. La obtención de la libertad, precisamente, había producido que estuvieran más desamparados. Al partir de cero, no disponían de refugio, ni comida y estaban sin alfabetizar; así las cosas, la mayor parte de ellos continuará al servicio de sus antiguos dueños. Sin olvidar la persecución de grupos racistas como el Ku Klux Klan. Por lo que la posibilidad de acceder al poder político, les permitiría ser considerados y protegidos por el gobierno (Davis, 2005: 78- 79).

La vía propuesta por Foster no obtuvo el mismo respaldo que la de Stanton, por lo que progresivamente se produjo una separación entre la lucha por los derechos de los/as afroamericanos/as y el movimiento sufragista e implicó agendas diferentes para cada movimiento, lo que representaba una falta de contacto, planteamiento y/o actividades conjuntas. Además, la aprobación de la Decimonovena Enmienda (1920), que permitía el voto femenino en Estados Unidos, al mismo tiempo, supuso una disminución de las reivindicaciones feministas, al considerar ésta como la única ruta para resolver la desigualdad de géneros.

4 Hermano de Harriet Beecher Stowe (escritora y famosa por su novela La cabaña del Tío Tom), fue un miembro destacado del movimiento abolicionista; también apoyó el sufragio femenino y estuvo inmerso en el movimiento por la templanza que criticaba el consumo de bebidas alcohólicas.

5 Abby Kelly Foster, junto a su marido Stephen Symonds Foster, lucharon por los derechos de las mujeres y la población negra. Fundadora de la Sociedad Americana Antiesclavista, colaboró con William Lloyd Garrison, editor del periódico abolicionista The Liberator. 


\section{La Segunda Ola: un único modelo de mujer.}

La inserción masiva en el mundo laboral -entendido como remunerado y público- o la entrada en las universidades hicieron posible la emancipación de las mujeres, quienes a pesar de poder votar y ser iguales ante la ley, seguían siendo discriminadas y relegadas a un segundo plano. La ampliación de la mirada feminista aportó un nuevo espectro al movimiento y especialmente implicó una nueva consciencia de la situación de desigualdad de las mujeres en todos los niveles y espacios de la vida. Esto se tradujo en la llamada Segunda Ola del feminismo -a partir de la década de los sesenta-, que diversificaba la agenda políitica e iniciaba los estudios sobre el patriarcado y los mecanismos de dominación del mismo. El lema «lo personal es político» de Kate Millett, en su obra Sexual Politics (1970), recoge a la perfección el carácter de esta etapa del feminismo (Reverter, 2012: 16).

El Segundo Sexo (1949) de Simone de Beauvoir y La mística de la feminidad (1963) de Betty Friedan fueron los dos textos más destacados, al hacer un recorrido por el patriarcado, cuestionar la división sexual del trabajo y realizar una revisión de la esfera pública y privada. No sólo se produjeron investigaciones aisladas, también el ámbito académico fue revisad, en especial los cánones epistemológicos, en los cuales se construía la investigación de cualquier rama científica. «El sujeto dominante (varón, heterosexual, blanco, occidental, burgués y de tradición y socialización judeo-cristiana)» (Reverter, 2012: 19) había marcado la identidad de la mirada de los estudios universitarios, por lo que se demandaba la entrada de una mayor diversidad identitaria en el discurso. La creación de nuevos estudios como los Gender Studies, los Black Studies o los Postcolonial Studies fue la respuesta, en un primer momento, en las universidades estadounidenses y británicas, a estas nuevas demandas (Reverter, 2012: 19-20).

Asimismo, el concepto de género fue una de las mayores aportaciones de esta revisión y marcó una nueva etapa en la investigación feminista. Al recoger este término un conjunto de características -sociales, culturales, religiosas, políticas, etcétera- asignadas y asumidas por cada individuo según su sexo (biológico), se consideraba que lo masculino está enfocado a los hombres y lo femenino a las mujeres. El género es una construcción sociocultural que implica variaciones dependiendo de la cultura, sociedad y momento histórico. Como se trata de un elemento puramente artificial es aprendido de manera consciente y subconsciente por medio de la educación, el lenguaje, la religión, el entorno social, etcétera (Reverter, 2012: 22-23).

La aparición de la Segunda Ola coincidiría con otros movimientos, especialmente en Estados Unidos con las luchas por los derechos civiles de la población negra. Al igual 
que durante el abolicionismo, las mujeres blancas vieron cierto paralelismo entre su situación y la de la población negra. En ambos casos, la vida cotidiana se veía afectada por la desigualdad y la discriminación, generando una brecha que los posicionaba en inferioridad respecto al hombre blanco. Además, la brutalidad policial contra las manifestaciones pacíficas -transmitida por los medios de comunicación-, dio impulso a la participación activa de la población blanca (especialmente de mujeres) en el movimiento por los derechos civiles.

La colaboración mixta se produjo a diferentes niveles, tanto dentro de las organizaciones y en las manifestaciones, como en el apoyo público y legal por parte de grupos y altos cargos políticos. Ejemplo de esto fueron la Asociación Nacional para el Progreso de la Gente de Color ${ }^{6}$-NAACP en sus siglas en inglés-, entre sus fundadores se encuentran dos blancos; o el Comité Coordinador Estudiantil No Violento (SNCC), organismo mixto que se destacó como principal impulsor de las sentadas y viajes de la libertad7 ${ }^{7}$. La elección de Stokely Carmichael en 1966 cambió esta dinámica, al expulsar ese mismo año a todos los integrantes blancos y dando paso a una etapa considerada más radical.

Sin embargo, la cooperación interracial no fue trasladada -al mismo nivel- en el movimiento feminista, e incluso se llegó a generar un distanciamiento entre ambos colectivos. Los motivos más destacados son la mayor trayectoria de las feministas blancas y las diferencias entre las reivindicaciones de las mujeres blancas y negras. La mayor trayectoria de la lucha feminista entre las mujeres blancas tiene un gran precedente en la llustración con Olympe de Gouges o Mary Wollstonecraft; así como un fuerte sentimiento de agrupación por medio del movimiento abolicionista -ya mencionado al inicio del presente artículo. Por el contrario, el feminismo negro «surgió en la confluencia (y tensión) entre dos movimientos, el abolicionismo y el sufragismo, en una difícil intersección» (Jarabo, 2012: 27), es decir, tuvo un siglo de retraso con respecto al blanco, una consecuencia de la esclavitud y la escasa formación de grupos feministas negros.

Además, la presión sobre la cuestión racial había sido una problemática de mayor importancia para las mujeres negras. Pero no se traduce en una falta de consciencia de

6 Asociación fundada en 1909 por Moorfield Storey, Mary White Ovington y William E. B. Du Bois. Su objetivo principal sigue siendo -aún hoy en día-garantizar la igualdad política, educativa, social y económica de todas las personas, así como eliminar el odio racial y la discriminación (NAACP. 2017).

7 Entre las actividades pacíficas realizadas por el movimiento, se encuentran las sit-in (sentadas), las freedom marches (marchas por la libertad) y las freedom riders (viajeros por la libertad). Esta última consistía en realizar viajes hacia estados sureños en autobuses mixtos y sin separación racial, una actividad prohibida por ley en estos estados. El recibimiento de estos autocares normalmente se saldó con la detención de los viajeros o, en el peor de los casos, con el asalto por parte de grupos racistas locales. 
su doble discriminación por ser mujer y negra, como demuestran Sojourner Truth ${ }^{8}$ e Ida B. Wells; ; aunque sí las convierte en excepciones, al ser de las primeras en denunciar su situación. Truth, con su discurso titulado Ain't I a woman? (¿No soy una mujer?) de 1852, ya reclamaba la participación de las afroamericanas en la lucha feminista (Davis, 2005: 69):

Los clubs de mujeres negras fueron excluidos; e incluso en las grandes marchas por el sufragio femenino, las líderes (blancas) del movimiento asumieron la política segregacionista instando a las mujeres negras a caminar de forma separada. Esta vivencia constante del racismo incluso entre las intelectuales negras de los grupos más acomodados sirvió, también desde el principio, como nexo de unión con las mujeres negras de clase trabajadora, creando un vínculo interclasista que ha diferenciado al feminismo negro del feminismo blanco de origen burgués (Jarabo, 2012: 32).

El rechazo por parte del feminismo blanco implicó un enfrentamiento entre colectivos, que se mantuvo durante la Segunda Ola. Y se vio reforzado por considerar como universal un único modelo de mujer en sus reivindicaciones, es decir, estaba dirigido por y para mujeres blancas de clase media-alta. Esto dificultaba la conexión entre colectivos, al tener ambos necesidades y problemáticas diferentes.

Además, el conflicto se vio reforzado por parte del movimiento por los derechos civiles, especialmente en su etapa final con el Black Power ${ }^{10}$ (1966). Una nueva línea de pensamiento que recelaba de la ayuda ofrecida por los blancos y que calificaba como traidores/ as a sus congéneres si decidían colaborar con alguna organización blanca. En respuesta a la crítica -que incluía al movimiento por los derechos civiles- por parte del feminismo, precisamente cuando la población negra había reforzado su unidad como comunidad entorno a las congregaciones religiosas que impulsaban valores y roles de género machistas. El siguiente punto profundizará en este pensamiento de rechazo del feminismo dentro del movimiento por los derechos civiles.

La participación activa de las afroamericanas dentro de las asociaciones por los derechos civiles les estaba ofreciendo una nueva visión de su propia comunidad y de sus representantes y comenzaban a ser conscientes de la necesidad de luchar por sus propias

8 Truth, nacida como esclava, obtuvo su libertad al escapar en 1826 junto a su hija. Se convirtió en activista abolicionista -participando directamente en el ferrocarril subterráneo- y posteriormente en sufragista. También fue la primera mujer negra en ganar un juicio contra un hombre blanco y así recuperar la custodia de su hijo.

9 Wells, sufragista y activista por los derechos de la población negra, centró su trabajo en la lucha contra los linchamientos. Sus publicaciones en periódicos, como el Chicago Tribune, y en panfletos denunciaban la violencia ejercida contra los afroamericanos. 10 Stokely Carmichael, durante un discurso en Greenwood (Mississippi) -el 16 de junio de 1966-, lanzó el grito We want Black Power! 
problemáticas. Un ejemplo fue Ella Baker ${ }^{11}$, crítica con la NAACP y la Conferencia de Dirigentes Cristianos del Sur ${ }^{12}$ (SCLC) por ser agrupaciones encabezadas por personajes carismáticos -como Martin Luther King-, quienes buscaban más los titulares que el trabajo diario interno. Además, estaba la exclusión de las mujeres dentro de la jerarquía y dirección de las asociaciones, compuestas principalmente por varones y líderes religiosos. Una situación que impidió a mujeres como Baker llegar a la directiva, quedando relegadas al trabajo de campo o como meras secretarias (o manos derechas de sus líderes).

La activista Pauli Murray ${ }^{13}$ recoge perfectamente la situación de las afroamericanas en ese momento, bajo el nombre de Jane Crow. La composición del término hace referencia a las Jim Crow, conglomerado de leyes racistas sureñas que impedían el contacto público y privado de la población blanca y negra y los/las consideraban como ciudadanos/as de segunda. Al sustituir Jim por Jane incluye la discriminación de la mujer negra, tanto por su raza como por su sexo.

La reducida trayectoria organizativa y la falta de concienciación entre las afroamericanas, así como los conflictos con el feminismo blanco, ocasionaron que no fuera posible hablar de Black Feminism (feminismo negro) hasta la Tercera Ola en la década de los noventa. Esto no implica una inexistencia de planteamientos feministas entre las afroamericanas, sino la tardía consolidación, reconocimiento y teorización del mismo por parte de esta comunidad. También la autocrítica del feminismo y la comprensión de la existencia de otras realidades que afectan a mujeres completamente diferentes entre sí, es decir, la urgencia de nuevos modelos de feminismo que respondan a las necesidades de cada una de ellas. «El feminismo negro, por lo tanto, fue desarrollado en parte para corregir inexactitudes históricas y oponerse a los estereotipos negativos de las mujeres negras» (Brown, 2001: 319 ).

\section{Jane Crow, mecanismos de control patriarcal}

Un factor clave para comprender la evolución del feminismo negro se encuentra en las experiencias personales de las mismas afroamericanas, así como en la necesidad de

\footnotetext{
11 Baker formó parte de la NAACP a partir de 1938, llegando a ser secretaria y directora de diversas ramas entre 1943 y 1946 , hasta obtener la presidencia de la sede de Nueva York en 1945. Pasó a formar parte de la SCLC como secretaria -sólo un año después de su formación-, así como coordinadora de campañas de 65 sedes. Esto la posicionaba como la mejor candidata para dirigir la organización, pero en el último momento fue cedida a Martin Luther King. En la siguiente década, se dedicó a asociaciones estudiantiles como la SNCC o la Mississippi Freedom Democratic Party, delegación del Democratic National Convention para la ayuda al voto negro (Langston, 2002: 15-17).

12 Fundada en 1957, como parte del boicot a los autobuses de Montgomery (Alabama), estuvo liderada por Martin Luther King hasta su muerte. Asimismo, agrupaba a diferentes líderes religiosos por la lucha de los derechos civiles.

13 Murray formó parte de la comisión de John F. Kennedy sobre la cuestión de la mujer en 1966. Al considerar que las mujeres necesitaban un organismo similar a la NAACP, cofundó ese mismo año la National Organization for Women (NOW). También fue crítica con el Black Power, rechazando a sus líderes por machistas (Langston, 2002: 160-161).
} 
romper con los estereotipos y los modelos impuestos hacia ellas. Por ello es necesario realizar una aproximación a su situación durante la década de los sesenta. El punto de partida consiste en una diferenciación entre patriarcado blanco y patriarcado negro, ambos implican un «sistema de dominación de las mujeres a todos los niveles y espacios» (Reverter, 2012: 16), pero diferenciado en su punto de origen. El primero impulsado por las instituciones gubernamentales y en general, la sociedad blanca; mientras que el segundo desde la comunidad negra y las asociaciones del movimiento por los derechos civiles. Ambos patriarcados se transformarán, responderán e incluso se complementarán dependiendo de la ideología y del momento histórico.

El patriarcado blanco operaba a diferentes niveles, destacándose el control sobre la familia y el cuerpo. Bajo la idea central de ser lo más blanco/a posible, ya que todo rastro de sus orígenes africanos -englobados bajo la denominación de negritud-era denostado. El paradigma era convertirse en el modelo blanco, el cual consistía en una familia nuclear y heterosexual, con un cabeza de familia varón y una madre entregada al hogar y al cuidado de los hijos. Un patrón impulsado incluso para la comunidad blanca, como recoge Friedan:

\begin{abstract}
Ser ama de casa en un barrio residencial era el sueño dorado de todas las jóvenes norteamericanas y la envidia, se decía, de las mujeres de todo el mundo. [...] Como amas de casa y madres respetadas en la misma forma que lo eran sus maridos en su mundo. Podían elegir libremente sus automóviles, sus trajes, sus aparatos electrodomésticos, sus supermercados; tenían todo lo que la mujer había soñado siempre. [...]

Quince años después de la segunda Guerra Mundial, esta mística de la perfección femenina se convirtió en el centro de la cultura contemporánea norteamericana. [...] Su único sueño era ser perfectas esposas y madres; tener cinco hijos y una hermosa casa; su única lucha, «pescar» y conservar un marido. No tenían ninguna opinión sobre los problemas no femeninos del mundo: deseaban que fuese el hombre el que tomara las decisiones importantes (Friedan, 1965: 32).
\end{abstract}

Las mujeres negras no sólo tenían como meta esta imagen de la mujer blanca como la perfección, también se las culpabilizó de la problemática social de su propia comunidad. El sociólogo E. Franklin Frazier o el historiador Kenneth Stampp habían planteado años antes la idea de un matriarcado negro. Pero fue Daniel P. Moynihan, con su The Negro Family. The Case of National Action (1965), quien impulsó definitivamente esta idea. El Informe Moynihan -como es más conocido- fue un encargo del presidente Lyndon B. Johnson para conocer los motivos que rodeaban a la situación de miseria y pobreza de la población negra (Jarabo, 2012: 43). 
Moynihan realiza un estudio comparativo entre las familias de ambas poblaciones. El modelo estándar utilizado es el de una unidad familiar nuclear-heterosexual; por consiguiente, el resto de modelos familiares pasaban a ser considerados familias desestructuradas, al ser incapaces de transmitir unos valores educativos y morales adecuados a sus hijos/as. Y en un factor (casi determinante) para desarrollar una tendencia delictiva, según recoge el Informe Moynihan. En esta categoría, entraban parejas divorciadas, familias monoparentales -mayoritariamente formadas por madres solteras-, hijos/as ilegítimos o no reconocidos.

El Informe presentaba una imagen estereotipada de las mujeres negras como madres controladoras -desde la esclavitud-, que criaban a futuros hombres sumisos e incapaces de valerse por sí mismos; por consiguiente, incapaces de formar y mantener una familia. Además, propone como solución el ingreso en el Ejército, como forma de alejamiento del control materno y endurecimiento del carácter:

There is another special quality about military service for Negro men: it is an utterly masculine world. Given the strains of the disorganized and matrifocal family life in which so many Negro youth come of age, the Armed Forces are a dramatic and desperately needed change: a world away from women, a world run by strong men of unquestioned authority, where discipline, if harsh, is nonetheless orderly and predictable, and where rewards, if limited, are granted on the bases of performance. The theme of current Army recruiting message states it as clearly as can be: «In the U.S Army you get to know what it means to feel like a man» (Moynihan, 1965: 42) 14 .

El aprendizaje de la autoridad masculina -es decir, del patriarcado- parece la idea que subyace tras esta propuesta, ya que considera que es precisamente esto el auténtico promotor para la prosperidad de la comunidad negra. «Sexism fosters, condines, and supports male violence against women, as well as encouraging violence between males. In patriarchal society, men are encouraged to channel frustrated aggression in the direction of those without power- women and children» (bell hooks, 1981: 105) 15 .

La idea de un matriarcado negro ha sido duramente criticada por el feminismo negro. The Black Woman's Role in the Community of Slaves (1972) de Angela Davis o Ain't I a woman. Black Women and feminism (1981) de bell hooks son dos de los estudios más destacados. Ambas autoras repasan los roles de género desde la esclavitud y rebaten la

\footnotetext{
14 Hay otra cualidad especial sobre el servicio militar para los hombres negros: es un mundo completamente masculino. Dadas las tensiones de la vida familiar desorganizada y matrifocal en la que tantos jóvenes negros alcanzan la mayoría de edad, las fuerzas armadas son un cambio dramático y desesperadamente necesario: un mundo alejado de las mujeres, un mundo dirigido por hombres fuertes de autoridad incuestionable, donde la disciplina, si es dura, no obstante, es ordenada y predecible, y donde las recompensas, aunque limitadas, se otorgan sobre la base del rendimiento. El mensaje de reclutamiento del ejército actual lo expresa claramente: «En el ejército de los Estados Unidos, se puede saber lo que significa sentirse como un hombre».

15 La escritora y activista feminista Gloria Watkins emplea el pseudónimo de bell hooks (en minúsculas) como homenaje a su abuela.
} 
existencia de un matriarcado. Sin embargo, el matriarcado negro fue aceptado por parte de la comunidad negra implicando una reafirmación de la masculinidad, que se trasladaba a un mayor control sobre las afroamericanas que pasaban a ser meros sujetos pasivos -bajo el rol de esposa y madre.

Además, los varones blancos, desde la esclavitud hasta la década de los sesenta, habían representado todo aquello a lo que se podía aspirar. Ellos se situaban en la cúspide de la pirámide social, al disponer de todas las libertades, estar protegidos por la ley y gozar de todos los derechos. Una cima sedimentada sobre la dominación racial, sexual, económica, etcétera, por consiguiente, se asoció a la idea de poder y éxito con una masculinidad controladora y represiva. Un concepto recogido por los afroamericanos: «The black militant's cry for the retrieval of black manhood suggests an acceptance of this stereotype, an association of masculinity with male dominance and tendency to treat the values of self-reliance and Independence as purely masculine traits» (Guy-Sheftall, 1995: 187). La supuesta imposibilidad de ejercer este poder sobre su entorno, al encontrarse en una comunidad supuestamente matriarcal, les suponía un sentimiento de castración.

El movimiento por los derechos civiles favoreció la unidad de la comunidad, en torno a la congregación religiosa. Al autoreforzarse por medio de estas instituciones se fortalecieron los modelos de familia y los roles de género tradicionales, como mecanismo de protección frente al racismo. Por lo tanto, no significa que fueran diferentes ambos patriarcados, sino que el negro tuvo como referente al blanco; el cual era cuestionado por el feminismo, mientras que el patriarcado negro se vio reforzado:

In this context, American Americans are counseled to accept traditional gender ideology's prescription of complementary gender roles for men and women (strength and weakness), and to believe that, although these gender roles may be more difficult for African American to attain, such roles are nonetheless natural and normal (Collins, 2005: 183) ${ }^{16}$.

El control sobre el cuerpo fue otra de las piezas claves, convirtiéndose en un tema incluso de carácter político. Henry Gates, en su libro Gente de color, recoge así la importancia del aspecto físico:

El pelo «bueno» era el lacio y el «malo» era el crespo. Incluso a finales de los años sesenta, en el apogeo del Black Power la mayoría de la gente no lograba obligarse a decir «malo» en lugar de «bueno» y «bueno» en lugar de «malo». Aún decían que el pelo como el de los blancos era el «bueno» [...] Todas las personas que yo conocía cuando era niño querían tener

16 En este contexto, se aconsejaba a los estadounidenses que aceptaran la prescripción tradicional de la ideología de género de roles de género complementarios para hombres y mujeres (fortaleza y debilidad), y que creyeran que, aunque estos roles de género pueden ser más difíciles de lograr para los afroamericanos, dichos roles son no obstante naturales y normales. 
el pelo «bueno». Podías ser feo como un demonio, estar sumido en la pobreza y, aún así, ser considerado atractivo, si tenías el pelo «bueno» [...].

Mi propio cabello «no era de lo peor», como decían los barberos la primera vez que me pelaban. Era como si un doctor informara de los resultados generales del primer examen físico completo que te ha hecho. «Estás en buena forma» o «la presión está un poco alta; hay que bajar un poco la sal» (Gates, 2013: 66-67).

Gates menciona el Black Power, una línea de pensamiento surgida a finales del movimiento, apoyada mayoritariamente por las generaciones más jóvenes. La pasividad de las organizaciones, la represión policial o el abandono por parte del gobierno, fueron algunas de las denuncias de esta nueva corriente. También apostaba por un mensaje de empoderamiento como comunidad, redescubriendo su historia y sus particularidades culturales y fisiológicas. La autodeterminación como afroamericanos/as resulta clave para comprender el fenómeno. Al aceptar el término, no sólo recordaban su origen africano -producto de la esclavitud-, sino también su presente como estadounidenses y por ello ciudadanos/as de plenos derechos. La revalorización del cuerpo, por medio de lemas como «Black is beautiful», o la aparición del estilo afro o natural no sólo eran cambios estéticos, también representaban un empoderamiento de sus rasgos como afrodescendientes.

Sin embargo, la ruptura con el patriarcado blanco no fue total, ya que siguió presente la idea de la mujer en segundo plano y la masculinidad negra como elemento a reforzar. En este punto, el patriarcado negro pasa a justificarse con el retorno a una supuesta tradición familiar africana, lo cual era una nueva excusa para la subyugación de las afroamericanas:

While the 60s black power movement was a reaction against racism, it was also a movement that allowed black men to overtly announce their support of patriarchy. Militant black men were publicly attacking the White male patriarchs for their racism but they were also establishing a bond of solidarity with them based on their shared acceptance of and commitment to patriarchy. The strongest bonding element between militant black men and White men was their shared sexism -they both believed in the inherent inferiority of woman and supported male dominance. Another bonding element was the black male's acknowledgement that he, like the White male, accepted violence as the primary way to assert power (bell hooks, 1981: 98-99)17.

\footnotetext{
17 Si bien el movimiento del poder negro de los años 60 fue una reacción contra el racismo, también fue un movimiento que permitió a los hombres negros anunciar abiertamente su apoyo al patriarcado. Los hombres negros militantes atacaban públicamente a los patriarcas blancos por su racismo, pero también establecían un vínculo de solidaridad con ellos sobre la base de aceptación y compromiso compartido con el patriarcado. El elemento de unión más fuerte entre los hombres negros militantes y los hombres blancos fue su sexismo compartido: ambos creían en la inferioridad inherente a la mujer y apoyaban el dominio masculino. Otro elemento de vinculación fue el reconocimiento del hombre negro de que él, al igual que el hombre blanco, aceptaba la violencia como la principal forma de ejercer el poder.
} 
El mantenimiento del matriarcado negro como un elemento real, y el posicionamiento del hombre negro como líder de la lucha contra el racismo y la desigualdad, muestran cómo el patriarcado no distingue entre razas, clase social, etc. Además, el acercamiento al feminismo se convirtió en una traición a la comunidad al ser considerado parte del patriarcado blanco, identificado por ellos como White Power (Cole y Guy-Sheftall, 2003: 92).

Las nuevas agrupaciones como el Black Panther Party ${ }^{18}$ (BPP) -en la etapa final del movimiento- recogieron la idea de un matriarcado negro, es decir, la necesidad de reforzar el papel de los hombres como elemento de ruptura contra el racismo y el control materno. Por ello, el inicio del BPP estuvo marcado por el impulso de una masculinidad agresiva -especialmente controladora de las mujeres-, manteniendo un papel secundario para sus compañeras e incluso, en algunos casos, sus líderes llegaron a considerarlas como herramientas contra el White Power.

En el caso del BPP, se produciría progresivamente una autocrítica sobre el papel de las mujeres entre sus filas, tanto a nivel personal (de sus líderes) como estructural. No se puede afirmar que el BPP se convirtiera en una organización feminista, pero sí fue una de las primeras en contar con un elevado número de líderes mujeres y luchar contra la desigualdad de las afroamericanas. Sin embargo, los diferentes frentes abiertos -tanto conflictos internos como externos, especialmente con el FBI-, la dificultad de realizar una autocrítica sobre el machismo en su comunidad y, posteriormente, la creación de nuevas vías de lucha contra el mismo, desde dentro de la organización, ocasionaron que no pudieran llegar realmente a profundizar en esta problemática.

La participación activa de las afroamericanas no sólo las enfrentó al racismo de la sociedad estadounidense, también al sexismo dentro de su propia comunidad. Pero la creciente conciencia de su doble discriminación (Jane Crow) se encontró desconectada del feminismo del momento. Éste estaba basado en un modelo de mujer blanca, heterosexual y de clase media-alta, por lo que sus problemáticas y reivindicaciones resultaban ajenas para las afroamericanas.

Además, la mayor trayectoria del feminismo entre las mujeres blancas contrastaba con la escasa concienciación entre las mujeres negras, quienes a consecuencia de la esclavitud $y$, posteriormente, de la falta de organizaciones que lucharan contra la doble discriminación se encontraban con un siglo de retraso. La falta de apoyo dentro de su misma comunidad no favoreció. Las décadas de los cincuenta y sesenta son, precisamente, un momento para

18 El Black Panther Party for Self-Defense -posteriormente conocido con un nombre más corto- nació en octubre de 1966 en Oakland (California) de manos de Bobby Seale y Huey P. Newton. La decisión de fundar este grupo surgió tras el estallido de una serie de disturbios en todo el país, respondidos con brutalidad policial. La idea era crear un grupo de vigilancia y de protección para la comunidad. 
reforzar modelos de familia y roles de género tradicionales, al ampararse en la unión en torno a la congregación como forma de protección frente al racismo.

Por ello, la creación de un feminismo propio -por y para las afroamericanas-, teo-

rizado, reconocido y consolidado, no se hallará hasta la década de los noventa. Entre los sesenta y los ochenta, se encuentran los inicios de esa creciente conciencia y se generan agrupaciones puramente enfocadas a la lucha contra el racismo y el sexismo que subyugan a las mujeres negras.

A partir de esta primera aproximación al tema que hemos planteado en este artículo, se abre toda una línea de investigación que esperamos poder explotar en un futuro para dejar constancia de una lucha sin parangón y cuyo aliento puede constituir una fuente de inspiración para nosotras, las mujeres de hoy en día.

\section{Bibliografía}

BELL HOOKS (1981). Ain't I a woman. Black Women and feminism, Boston: South End Press.

Brown, Kimberly N. (2001). "Divas revolucionarias: feminismo negro de los sesenta hasta nuestros días» en Driscoll de Alvarado, Bárbara y Paz Consuelo Márquez-Padilla (coord.) (2001). El color de la tierra: minorías en México y Estados Unidos, México D.F.: Universidad Nacional Autónoma de México, pp. 3 17-332.

Cole, Johnetta Bestcha y Beverly Guy-Sheftall (2003). Gender Talk. The Strugg/w for Women's

Equality in African American Communities, Nueva York: Ballantine Books.

Davis, Angela Y. (2005). Mujer, raza y clase, Móstoles (Madrid): Akal.

FrIEDAN, Betty (1965). La mística de la feminidad, Barcelona: Sagitario.

Gates, Henry L. (2013). «La cocina» en Gates, Henry L. (2013). Gente de color, La Habana:

Editorial Arte y Literatura, pp. 63-73.

GuY-SHEFTALL, Beverly (1995). Words of fire: an anthology of African-American feminist thought,

Nueva York: New Press.

JABARDO, Mercedes (ed.) (2012). Feminismos negros. Una antología. Madrid: Traficantes de sueños. LANGSTON, Donna (2002). A to Z of American women leaders and activists. Nueva York: Facts On File.

Morniman, Daniel P. (1965). The Negro Family. The Case of National Action, Washington D.C.: Office of Policy Planing and Research United States Departement of Labor. Disponible en: https://web.stanford.edu/ mrosenfe/Moynihan\%27s\%20The\%20 Negro\%20Family.pdf (Fecha de consulta: 26/1/2018). 
NAACP (2018). «NAACP: 100 years of History», en NAACP. Disponible en: http://www. naacp.org/pages/naacp-history (fecha de consulta: 12/4/2018).

ReVerter Bañón, Sonia (2012). «Los estudios de género» en Torrent, Rosalía y Sonia Reverter (eds.), Variaciones sobre el género, Castellón: ACEN, pp. 15-31.

Stanton, Elizabeth Cady; Anthony, Susan B. y Matilda Joslyn GaCe (eds.) (1887). History of woman suffrage, Vol. 2 (1861-1876), Rochester (Nueva York): Charles Mann.

Recibido el 5 de octubre de 2018 Aceptado el 23 de diciembre de 2018 BIBLID [1 139-1219 (2018) 24: 109-124] 